Research Paper

\title{
Impact of Patient Navigation from Diagnosis to Treatment in an Urban Saiety Net Breast Cancer Population
}

\author{
Nisreen A Haideri ${ }^{\bowtie}$, Jill A Moormeier \\ 1. The University of Kansas Cancer Center, Westwood KS and Kansas City Veterans Administration Medical Center, Kan- \\ sas City, MO, USA \\ 2. University of Missouri School of Medicine, 2411 Holmes, Kansas City, MO 64108, USA
}

$\triangle$ Corresponding author: Nisreen A Haideri MD, 2330 Shawnee Mission Parkway Suite 210, Westwood KS 66205. Telephone: 913-588-6077; Fax: 913-588-4085; Email: nhaideri@kumc.edu

(C) Ivyspring International Publisher. This is an open-access article distributed under the terms of the Creative Commons License (http://creativecommons.org/ licenses/by-nc-nd/3.0/). Reproduction is permitted for personal, noncommercial use, provided that the article is in whole, unmodified, and properly cited.

Received: 2011.06.16; Accepted: 2011.09.03; Published: 2011.09.08

\begin{abstract}
Background: Disparities between U.S. population groups in cancer incidence, treatment and outcome have been well documented. Literature evidence is scarce regarding the impact of patient navigator programs on elimination of these differences.

Methods: This is a retrospective case series analysis. The pre -navigation group included patients diagnosed between January I, 1997 and December 3I, 1999. The post -navigation group included patients diagnosed between January I, 2000 and December 3I, 2003. Cancer stage, time from presentation to treatment and treatment outcome were compared by review of medical records.

Results: Three hundred and thirty five women were diagnosed between January I, I 997 and December 3I, 2003. Thirteen patients were ineligible, I03 women in the pre- navigation group, and 219 women in the post-navigation group. 157 (72\%) received navigation services. The median time to first treatment was decreased by 9 days ( 42 days in pre -navigation group compared to 33 days in post -navigator group). Race, insurance and clinical presentation did not influence the time to treatment.

Conclusions: Navigation program did not influence the stage of presentation or the overall survival of women. There was a modest decrease in the time between initial presentation and definitive therapy. The utility of navigator programs is likely to vary with each institution.
\end{abstract}

Key words: Patient Navigation, breast cancer, survival, underserved population

\section{Introduction}

Disparities between U.S. population groups in cancer incidence, treatment and outcome have been well documented. [1-4] Most commonly, racial and socioeconomic variations in cancer outcomes have been investigated. African-Americans, in particular, are disproportionately affected by the morbidity and mortality of neoplastic diseases, with higher cancer incidence rates among men and higher cancer mortality rates among both men and women than that observed in other population groups. [2,5] In addition, population measures of socioeconomic status have also been associated with cancer outcomes. People living in economically disadvantaged areas of the country have been consistently shown to have a higher cancer-associated mortality. [6-8]

Socioeconomic and racial or ethnic disparities in cancer outcomes can be partially explained by a number of factors including health care access, quality 
of care, the presence of co-morbid conditions, and tumor or host biology. While we are acquiring a better understanding of why disparities in cancer outcomes exist, there is very little information about effective means of eliminating those differences.

An increasingly popular approach to improving cancer care outcomes has been navigator programs. The first patient navigator program was introduced by Dr Harold Freeman, a breast surgeon at the Harlem Hospital in New York City. He observed a pattern of late stage presentation in breast cancer patients where the primary barrier to early, effective treatment was the inability to access medical care in a timely manner. The Patient Navigation program at Harlem Hospital was designed to overcome this barrier and the success of his efforts resulted in the establishment of patient navigation programs throughout the country. [9-11]

Patient navigators are members of the community who have a clear understanding of the local social and cultural issues and are simultaneously aware of the functioning of the health care system. They have established contacts with the medical providers, particularly specialists in surgery, radiation oncology, medical oncology and radiology, as well as with the hospital support services such as social work and financial counseling. The most important role of patient navigation is to assist an individual with a suspicious cancer-related finding to receive a timely diagnosis and treatment. The navigator accomplishes this most effectively through one-on-one contact with the patient from the time of initial suspicion of cancer diagnosis. This process is intended to eliminate barriers to diagnosis and treatment. [9] While this was the primary focus of our Patient Navigators, many different models of patient navigation are being employed across the country, having very different goals. Many are hospital based programs focused on improving clinical care and patient satisfaction in the hospital setting. They might not necessarily focus towards improving health disparities. [11]

While patient navigation programs have spread throughout the country, their impact upon cancer outcomes is understudied. [11,12] Through the evaluation of the processes and outcomes of breast cancer care at a safety net hospital over the time that a navigator program was adopted, this study attempts to better define the impact of such programs.

\section{Materials and Methods}

Patient Population. All women with adenocarcinoma of the breast diagnosed at Truman Medical Center in Kansas City, Missouri between January 1, 1997 and December 31, 2003 were eligible for inclu- sion in this study. The only women excluded from analysis were those who received their primary therapy elsewhere $(n=7)$.

Study Design. This was a retrospective case series analysis. Truman Medical Center is the safety net hospital for Kansas City. With the generous assistance of the American Cancer Society, a Navigator program began serving patients in January 2000. Eligible women with a breast cancer diagnosed between January 1, 1997 and December 31, 1999 were considered the standard management group. Women with breast cancer diagnosed between January 1, 2000 and December 31, 2003 were considered the Patient Navigator group.

Intervention. The Patient Navigator program included three health care facilitators. Women were identified by their treating providers and offered enrollment in the program at or around the time of their breast cancer diagnosis. This was most often at the time of presentation to the Breast Clinic for evaluation of an abnormal mammogram or palpable breast mass. Participation was entirely voluntary.

Women who chose to participate in the Navigator program were provided with added assistance in scheduling appropriate diagnostic and consultative services, arranging transportation and child care for scheduled appointments, access to a large collection of educational materials, and one-on-one emotional support through the initial diagnosis and treatment process. Subjects who chose to be navigated were provided with cell phones for appointment reminders, when needed. The navigators followed them on their subsequent clinic visits to promote adherence with treatment recommendations and help with daily interferences with treatment including providing cab passes for transportation and child care services. They acted as advocates through the process necessary to obtain financial assistance with health care expenses (Medicaid and hospital/clinic discount applications). Finally, they often served as an informal contact between patients and other health care providers, asking for more information, reporting changes in clinical status and treatment toxicities.

Data Collection and Analysis. Information on each patient was obtained by review of their medical record and Tumor Registry entry. Demographic data, tumor histology and staging, cancer treatment information and patient outcome data were obtained. In addition, a detailed timeline of cancer care was constructed. The date of first evaluation was defined as: 1) the date that the patient first contacted the health care system with a concern that led to the diagnosis of breast cancer (this was most commonly a visit to a primary care provider with a palpable breast abnor- 
mality), or 2) the date of an abnormal screening exam (most often mammogram) for clinically asymptomatic tumors. The date of first treatment was defined as the date that the patient began definitive therapy for the cancer, typically the date of a therapeutic surgical procedure or, if surgery was not the initial therapy, the date that chemotherapy or hormonal therapy was begun.

The primary outcome evaluated in this study was mean time to definitive therapy, defined as the difference between the date of first evaluation and the date of definitive therapy. Secondary outcomes were the proportion of women receiving definitive therapy within 60 days of clinical presentation and the time from surgery to beginning adjuvant therapy. All statistical analyses were performed with SPSS for Windows software (SPSS 16.0, SPSS, Chicago, IL). Descriptive data were compared using $X^{2}$ test. A two tailed $p$ value of $\leq 0.05$ was considered significant for all tests. Time to event analysis was performed using the Kaplan-Meier method and compared with the log-rank test. The impact of potentially confounding variables was considered using Cox regression analysis.

With a two sided a of 0.05 and an expected sample size of 300 patients, the study had a power of 0.85 to detect a five day difference in the primary outcome. The power was 0.80 to detect a $15 \%$ difference in the proportion of women receiving definitive therapy in 60 days.

\section{Results}

Three hundred thirty five women with adenocarcinoma of the breast were diagnosed between January 1, 1997 and December 31, 2003. Seven patients received their cancer treatment elsewhere and the first date of clinical contact for a breast cancer related sign or symptom could not be clearly determined in an additional six patients. These thirteen patients were excluded from further analysis, leaving a final study population of 322 women; 103 women in the pre navigator group, and 219 women in the post navigator group. Of the 219 women in the patient navigation group, 157 (72\%) accepted and received navigation services.

Table 1 outlines the demographic and tumor characteristics of the women studied.

The women in this study had a slightly lower median age, 55 years, than observed nationally and were evenly divided between Caucasian and African American race. One remarkable difference between the two study groups was the proportion of uninsured women, which was much lower during the period of time when patient navigation was available.
This decrease in the number of uninsured women was accounted for by a similar increase in the number of women with Medicaid insurance in the navigated group. Insurance status was measured six months after diagnosis (at the time of case accession in the Tumor Registry) and, thus, does not represent a difference in patient population at the time of diagnosis but, rather, may represent one potential impact of the navigator program.

Table I. Patient characteristics

\begin{tabular}{|c|c|c|c|}
\hline & $\begin{array}{l}\text { Standard } \\
\text { Management } \\
(n=103)\end{array}$ & $\begin{array}{l}\text { Navigation } \\
\text { Available } \\
(\mathrm{n}=219)\end{array}$ & $\begin{array}{l}\text { Combined } \\
\text { Population } \\
(\mathrm{n}=322)\end{array}$ \\
\hline Median Age & 54 years & 56 years & 55.5 years \\
\hline Race & $\%$ & $\%$ & $\%$ \\
\hline White & 41.7 & 53.4 & 49.7 \\
\hline Black & 55.3 & 44.3 & 47.8 \\
\hline Other & 2.9 & 2.4 & 2.4 \\
\hline \multicolumn{4}{|l|}{ Clinical Presentation } \\
\hline Breast mass & 27.7 & 45.2 & 39.9 \\
\hline Abnormal mammogram & 67.0 & 45.2 & 51.8 \\
\hline Unknown & 5.3 & 9.7 & 8.3 \\
\hline \multicolumn{4}{|l|}{ Insurance } \\
\hline Commercial insurance & 7.8 & 11.0 & 9.9 \\
\hline Medicare & 35.9 & 28.8 & 31.1 \\
\hline Medicaid & 17.5 & 49.8 & 39.4 \\
\hline Uninsured & $37.9 *$ & 9.6 & 18.6 \\
\hline Other & 1.0 & 0.9 & 0.9 \\
\hline \multicolumn{4}{|l|}{ Histology } \\
\hline Invasive ductal & 79.6 & 72.6 & 74.8 \\
\hline Invasive lobular & 2.9 & 3.7 & 3.4 \\
\hline Carcinoma-in-situ & 9.7 & 7.3 & 8.1 \\
\hline Others & 6.9 & 16.7 & 12.7 \\
\hline \multicolumn{4}{|l|}{ Stage } \\
\hline 0 & 10.7 & 14.6 & 13.4 \\
\hline I & 24.3 & 23.7 & 23.9 \\
\hline IIA & 29.1 & 23.3 & 25.2 \\
\hline IIB & 14.6 & 15.5 & 15.2 \\
\hline IIIA & 8.7 & 7.8 & 8.1 \\
\hline IIIB & 5.8 & 4.1 & 4.7 \\
\hline IIIC & 0 & 0.9 & 0.6 \\
\hline IV & 5.8 & 10.0 & 8.7 \\
\hline \multicolumn{4}{|l|}{ Estrogen Receptor } \\
\hline Positive & 46.6 & 57.6 & 54.1 \\
\hline Negative & 23.3 & 23.5 & 23.4 \\
\hline Unknown/CIS & 30.1 & 18.9 & 22.5 \\
\hline \multicolumn{4}{|l|}{ Progesterone Receptor } \\
\hline Positive & 41.7 & 50.7 & 47.8 \\
\hline Negative & 27.2 & 29.5 & 28.8 \\
\hline Unknown/CIS & 31.1 & 19.8 & 23.5 \\
\hline
\end{tabular}


The navigators were expected to help and guide subjects with Medicaid and/or Medicare applications and with local arrangements for financial assistance *. There was no substantive difference in tumor characteristics in the two groups of women. The majority of tumors were infiltrating ductal carcinomas. The final pathologic stage is very similar between the two groups. Overall, $13 \%$ of women had carcinoma-in-situ. Of the 279 women with invasive cancer, $49 \%$ had local disease, $41 \%$ regional disease, and $10 \%$ distant disease.

The time from initial presentation, either the date of an abnormal screening test or the date of initial evaluation of a breast mass, to the date of definitive therapy was shorter during the period of time when patient navigation was available. The median time to first treatment was 9 days shorter (42 days compared to 33 days) after the institution of the navigator program. There was a much more dramatic difference in the mean values (70 days, 95\% CI (47-94) in the pre navigator group compared to 48 days, $95 \%$ CI (41-55) in the post navigator group, $\mathrm{p}=0.006$ ). The mean values, however, were strongly influenced by a very small number of women who chose to delay therapy for months (and in two cases, years) after initial presentation. Overall, $67 \%$ of women in the pre navigator group received definitive therapy within 60 days of initial presentation; $75 \%$ of women in the post navigator group had received definitive therapy within 60 days of presentation (Figure 1). For the large majority of women in both groups, the initial definitive therapy was surgery: in the standard therapy group $6 \%$ of women received systemic therapy (chemotherapy or hormonal therapy) as their initial treatment, in the navigation group $16 \%$ of women received systemic therapy as their initial treatment. For most women, the choice to use systemic therapy as initial treatment was made in the setting of distant metastatic disease. In the later time period, a small number of women with earlier stage cancer also received systemic therapy as their first treatment.

The only other pre-treatment variable significantly associated with time between clinical presentation and treatment was patient age $(p=0.02)$, with younger women experiencing a greater delay in therapy. Race, insurance type, and mode of clinical presentation were not associated with time to treatment. Multivariable regression analysis confirmed the independent association of both navigator availability $(p=0.04)$ and patient age $(p=0.02)$ in time to initial treatment.

The median time from surgery to the beginning of adjuvant therapy was the same in both groups, 40 days. The mean times were, likewise, very similar: pre navigator group, 48 days (95\% CI 39-56), post navigator group, 43 days (95\% CI 38-48), $\mathrm{p}=0.62$.

Overall survival was not influenced by the patient navigation program (Figure 2), as would be expected, given the relatively minor impact of the program upon evaluation and management time.

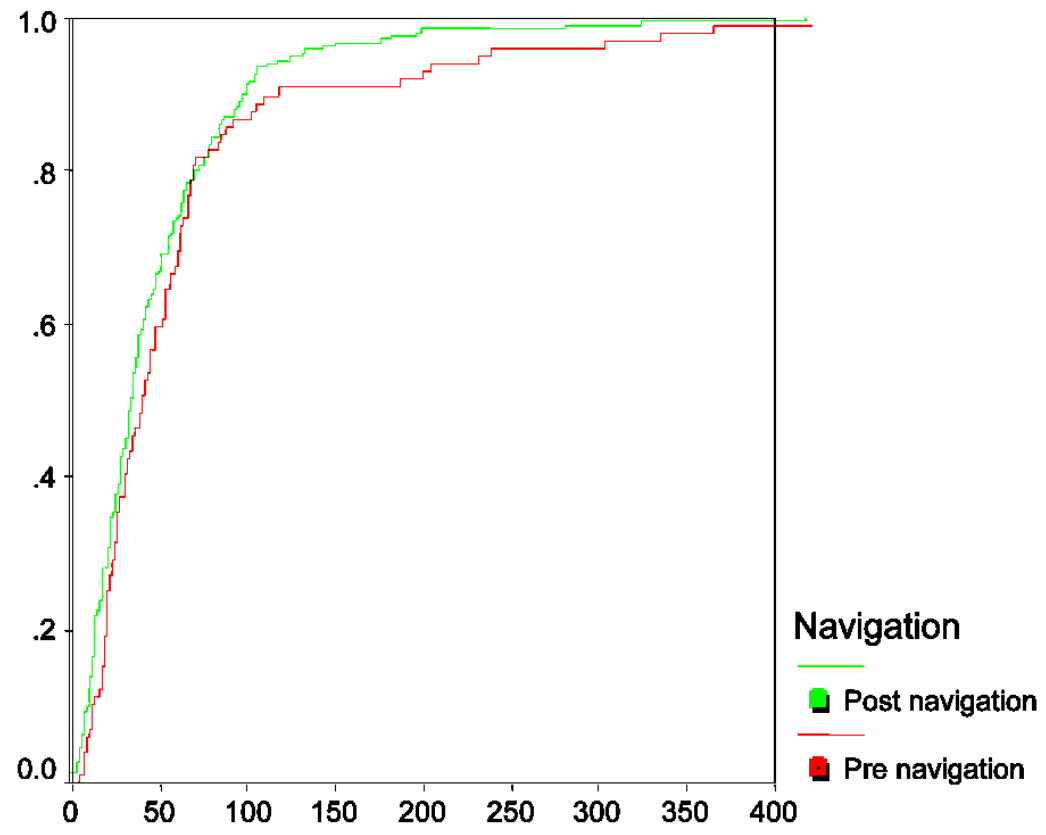

Time (days)

Figure I. Time to Definitive Therapy for Breast Cancer 


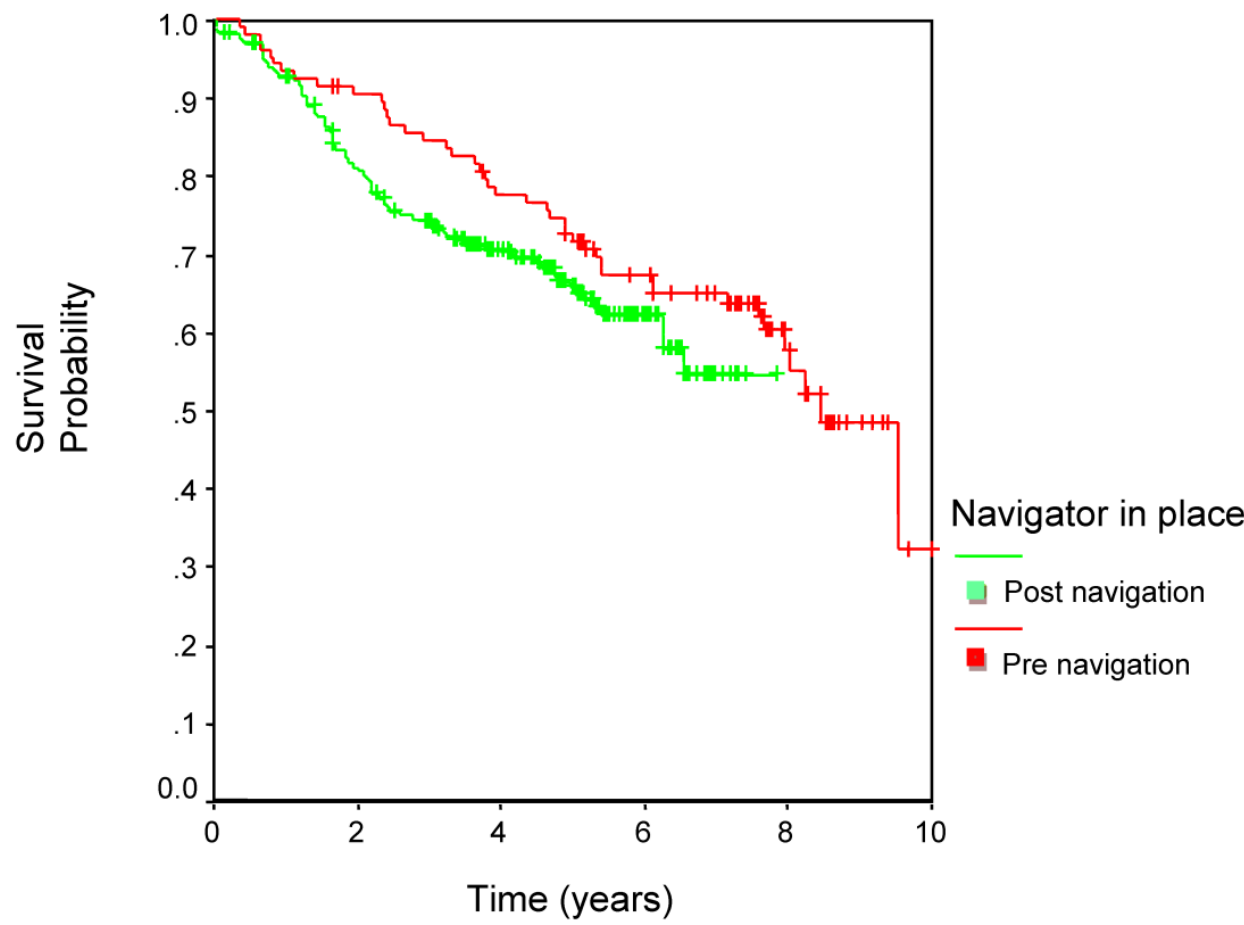

Figure 2. Survival.

\section{Discussion}

Implementation of a patient navigation program at this safety net hospital had a very modest effect on the time from symptom presentation to first treatment, decreasing the median time by 9 days, from 42 to 33 days. This study focused on processes of care from the time of identification of a suspicious finding through treatment of the cancer. We were unable to obtain accurate data on the first step in formal health care access - time to access of primary care, although the wait time for new patient appointments at our local community health centers and hospital based clinics is typically less than one month, suggesting that navigation to improve access earlier in the process of care would have also had very limited impact upon the early course of care.

Not surprisingly, given the relative ease of access to specialty care, patient navigation was not shown to have any influence on stage of disease at presentation or survival of breast cancer patients. The women in this study had more advanced breast cancer at presentation than that observed in the U.S. population as described by the SEER data over a similar time period.[13] Local disease was observed in 49\% (compared with $61 \%$ nationally), regional disease in $41 \%$ (31\% nationally) and distant disease in $10 \%$ (6\% nationally). This more advanced disease, primarily manifested by a shift from local to regional disease, may be the result of limited access to or use of primary care, lower routine breast cancer screening among our patient population, or biologically more aggressive disease. It does not; however, appear to be a marker of inability to successfully use the variety of services available for the diagnosis and treatment of cancer.

One significant difference in the population that received navigation services was the shift from complete lack of health insurance to enrollment in Medicaid. This variable was measured six months after diagnosis, not at the time of presentation. It is certainly possible that interaction with the patient navigator was influential in patients successfully applying and receiving Medicaid. This is a complex process requiring careful adherence to data requests and close follow-up. However, this analysis covered a time period during which the hospital implemented a variety of programs designed to increase Medicaid enrollment and these concurrent events likely confound the interpretation of the insurance data.

The definition of patient navigation varies between institutions. [11] It is generally recognized to be a barrier focused intervention provided to individual patients to assist with accessing cancer related care. [14] Culturally sensitive education and psychosocial support are frequently combined with case management functions, including careful testing follow-up, appointment scheduling, and care coordination. [15] 
The navigation function typically is limited to a set of services required to complete a component of cancer care and, therefore, distinguishes itself from traditional case management and social services functions. Further, the focus of navigation programs has been on those members of the community felt to be the most vulnerable to adverse disparities in cancer care and outcomes. While patient navigation programs reported in the medical literature employ a variety of techniques to provide improved access and elimination of barriers, the majority tend to focus on one area of cancer care: screening for malignancy and the activities surrounding this event. [14, 16-20] Both adherence to recommended screening guidelines (for breast, colorectal, cervical, and prostate cancer) and timely follow-up and resolution of screening abnormalities have been examined. Our study is one of the few to investigate the effect of patient navigation on the course of care and clinical outcome of women with a cancer diagnosis. Only the report of the original program from the Cancer Control Center of Harlem included a similarly large number of women with cancer. [10] In that study, a dramatic shift in breast cancer stage at diagnosis was observed after the implementation of a comprehensive cancer management and outreach program that included navigator services. The investigators saw the proportion of women with Stage III/IV breast cancer at diagnosis decrease from $49 \%$ prior to program implementation to $21 \%$ after program implementation. In our study, a similar shift to earlier stage disease could not be documented (Stage III/IV disease in the usual management group $-20.3 \%$, in the navigation group $-22.8 \%$ ). This could be explained as navigation began at the time of diagnosis, which is too late in the process to influence outcomes, such as stage of diagnosis.

The primary weakness of this study is the non-randomized, historical control, study design, leaving results open to differential outside influences. We expect that this explains the difference in the proportion of subjects covered by the state Medicaid program, as described earlier. There were no other obvious changes in the local or regional health care structure that would have been expected to influence our observations. A potential strength of this research design was the opportunity to observe the impact of the Patient Navigator Program on all women with breast cancer at the institution, rather than solely those agreeing to participate in the program. While a more favorable effect of the navigator program may have been observed if the analysis were limited to those who took advantage of the navigator services, this likely would represent women expected to have better outcomes as a result of their demonstrated ac- tive involvement in pursuit of medical care. By studying the impact of a navigator program in all women with breast cancer at a single institution, we potentially provide a more accurate description of the effectiveness of similar programs on the larger population.

Understanding that participation in the navigation program was voluntary and there could have been a selection bias from a subject prospective in the population studied in the post navigation era. Our aim was to look at the overall impact of patient navigation in this community based program, where it was established to help subjects have an easy access to barriers to care.

Data collection was limited to cancers diagnosed and treated at the host institution. To the best of author's capacity all eligible persons with cancer were captured and included in the study. If patients sought treatment at other hospitals, they were excluded from the study as mentioned earlier. As this was a retrospective analysis, other sociodemographic factors which can also influence treatment related barriers such as spouse and family support, living alone, education, income level, employment at the time after diagnosis were not available at the time of the study.

Improvement in overall survival with navigation intervention at the time of clinical presentation could not be expected as several factors including stage, biology of the disease, treatment received all play a major role in affecting survival. Our intention in this study was to evaluate the timeliness of care provided, as one possible factor influencing survival. However as overall survival is determined by many patient and disease characteristics, including stage, prognostic factors, and treatment provided, patient navigation would most likely not influence overall survival in this patient population without substantially affecting timeliness of care.

Patient navigation in the context of the activity studied in this analysis also included collaboration with the local hospital-based clinics and community health centers to facilitate patient access to specialty diagnostic and treatment services, direct patient contact to assist with education and information, coordination of consultations and diagnostic testing, assistance with transportation and communication, and psychosocial support over the course of cancer care. Review of the data from this study, when considered in the context of the available medical literature, suggests that maximum benefit from navigation programs will only be observed if characteristics of the local health care setting are carefully considered.

Programs that focus on easing access to cancer diagnostic and treatment services may be quite useful 
and effective in some settings, whereas other communities may benefit far more from community outreach programs that emphasize routine use of primary care and cancer screening services. It is very unlikely that a single programmatic approach to the observed disparities in cancer outcomes will be equally effective in all settings across this country.

\section{Acknowledgements}

We would like to thank Christine McReady and Seth Katz for help with data acquisition, Bennie Turner for information about the Patient Navigator Program at Truman Medical Center.

This study was presented at the Annual ASCO Meeting Orlando (May 28-June 1, 2009), at the General Poster Session in Health Services Research on May 30, 2009, 2-6 pm.

\section{Conflict of Interest}

The authors have declared that no conflict of interest exists.

\section{References}

1. Smedley BD, Stith AY, Nelson AR: Unequal treatment: Confronting racial and ethnic disparities in health care. Washington DC: Institute of Medicine, National Academies Press. 2003.

2. Edwards BK, Brown ML, Wingo PA, et al: Annual report to the nation on the status of cancer, 1975-2002, featuring population-based trends in cancer treatment. J Natl Cancer Inst 2005;97:1407-27

3. Shavers VL, Brown ML: Racial and ethnic disparities in the receipt of cancer treatment. J Natl Cancer Inst 2002;94:334-57

4. Bach PB, Schrag D, Brawley OW, et al: Survival of blacks and whites after a cancer diagnosis. JAMA 2002;287:2106-13

5. Newman LA, Griffith KA, Jatoi I, et al: Meta-analysis of survival in African American and white American patients with breast cancer: Ethnicity compared with socioeconomic status. J Clin Oncol 2006;24:1342-9

6. Singh GK, Miller BA, Hankey BF, et al: Changing area socioeconomic patterns in U.S. cancer mortality, 1950-1998: Part I-All cancers among men. J Natl Cancer Inst 2002;94:904-15

7. Singh GK, Miller BA, Hankey BF: Changing area socioeconomic patterns in U.S. cancer mortality, 1950-1998: Part II-Lung and colorectal cancers. J Natl Cancer Inst 2002;94:916-25

8. Boyd C, Zhang-Salomons JY, Groome PA, et al: Associations between community income and cancer survival in Ontario, Canada, and the United States. J Clin Oncol 1999;17:2244-55

9. Freeman, HP: A model patient navigator program. Oncol Issues 2004;19:44-47

10. Oluwole SF, Ali Ao, Adu A, et al: Impact of cancer screening program on breast cancer stage at diagnosis in a medically underserved urban community. J Am Coll Surg 2003;196:180-188

11. Dohan, D, Schrag D: Using navigators to improve care of underserved patients. Cancer 2005;104:848-855

12. Freeeman HP, Muth BJ, Kerner JF: Expanding access to cancer screening and clinical follow up among the medically underserved. Cancer Practice 1995;3:19-30

13. [Internet] Ries LAG, Melbert D, Krapcho M, Stinchcomb DG, Howlader N, Horner MJ, Mariotto A, Miller BA, Feuer EJ, Altekruse SF, Lewis DR, Clegg L, Eisner MP, Reichman M, Edwards BK. SEER Cancer Statistics Review, 1975-2005, National
Cancer Institute. Bethesda, MD. http://seer.cancer.gov/csr/ 1975_2005/

14. Wells KJ, Battaglia TA, Dudley DJ, et al: Patient navigation: state of the art or is it science? Cancer 2008;113:1999-2010

15. Till JE: Evaluation of support groups for women with breast cancer: importance of the navigator role. Health Qual Life Outcomes 2003;1:16

16. Ell K, Vourlekis B, Lee PJ, et al: Patient navigation and case management following an abnormal mammogram: a randomized clinical trial. Preventive Medicine 2007;44:26-33

17. Ferrante JM, Chen PH, Kim S: The effect of patient navigation on time to diagnosis, anxiety, and satisfaction in urban minority women with abnormal mammograms: a randomized controlled trial. J Urban Health 2007;85:114-24

18. Ell K, Padgett D, Vourlekis B, et al: Abnormal mammogram follow-up. A pilot study in women with low income. Cancer Practice 2002;10:130-8

19. Battaglia TA, Roloff $K$, Posner MA, et al: Improving follow-up to abnormal breast cancer screening in an urban population. A patient navigation intervention. Cancer 2007; 109(2 Suppl):359-67

20. Nash D, Azeez S, et al. Evaluation of an intervention to Increase Screening Colonoscopy in an Urban Public Hospital. J of Urban Health. 2006;83(2): 231-243 